UCRL-ID-121968

\title{
Monte Carlo Simulations of Solid-State Photoswitches
}

P.W. Rambo

J. Denavit

RECEIVED

NOV _ 71995

OSTI

September 1995

This is an informal report intended primarily for internal or limited external distribution. The opinions and conclusions stated are those of the author and may or may not be those of the Laboratory.

Work performed under the auspices of the U.S. Department of Energy by the Lawrence Livermore National Laboratory under Contract W-7405-Eng-48. 


\section{DISCLAIMER}

This document was prepared as an account of work sponsored by an agency of the United States Government. Neither the United States Government nor the University of California nor any of their employees, makes any warranty, express or implied, or assumes any legal liability or responsibility for the accuracy, completeness, or usefulness of any information, apparatus, product, or process disclosed, or represents that its use would not infringe privately owned rights. Reference herein to any specific commercial product, process, or service by trade name, trademark, manufacturer, or otherwise, does not necessarily constitute or imply its endorsement, recommendation, or favoring by the United States Government or the University of California. The views and opinions of authors expressed herein do not necessarily state or reflect those of the United States Government or the University of California, and shall not be used for advertising or product endorsement purposes.

This report has been repoduced directly from best available copy

Available to DOE and DOE contractors from the Office of Scientific and Technical Information P. O. Box 62, Oak Ridge, TN 37831

Prices available from (615) 576-8401, FTS 626-8401

Available to the public from the National Technical Information Service

U. S. Department of Commerce 5285 Port Royal Rd. Springfield, VA 22161 
LDRD FY'93 Final Report: Tracking Code \# 92-SR-012

Monte Carlo Simulations of Solid-State Photoswitches

\author{
P.W. Rambo and J. Denavit \\ University of California, Lawrence Livermore National Laboratory \\ P. O. Box 808, Livermore, CA 94551
}

\begin{abstract}
Large increases in conductivity induced in GaAs and other semiconductors by photoionization allow fast switching by laser light with applications to pulse-power technology and microwave generation. Experiments have shown that under high-field conditions (10 to $50 \mathrm{kV} / \mathrm{cm}$ ), conductivity may occur either in the linear mode where it is proportional to the absorbed light, in the "lock-on" mode, where it persists after termination of the laser pulse or in the avalanche mode where multiple carriers are generated. We have assembled a self-consistent Monte Carlo code to study these phenomena and in particular to model hot electron effects, which are expected to be important at high field strengths. This project has also brought our expertise acquired in advanced particle simulation of plasmas to bear on the modeling of semiconductor devices, which has broad industrial applications.
\end{abstract}

\title{
INTRODUCTION
}

Laser-triggered solid-state switches, operating at high fields have promising applications to fast pulse-power technology, to microwave generation, and may play an important role in the development of impulse radar. Experiments have been done with GaAs chips $2 \mathrm{~cm} \times 0.5 \mathrm{~cm}$ and $0.1 \mathrm{~cm}$ thick inserted in a parallel-plate transmission line having a gap of $0.5 \mathrm{~cm}$, a width of $2 \mathrm{~cm}$ and a length of approximately $1 \mathrm{~m}$. These chips are irradiated with $100 \mathrm{ps}$ laser pulses at $1.06 \mu \mathrm{m}$ wavelength and diagnostics along the line record the resulting electromagnetic (EM) pulse. Three modes of operation have been found experimentally [1].

(i) The linear mode in which each absorbed photon creates a conduction electron. In this mode, the switch closing time is limited only by the laser rise time and the opening time depends on carrier recombination. Operation in this mode has been achieved with fields up to $\approx 25 \mathrm{kV} / \mathrm{cm}$. The linear mode is easily understood theoretically, but would yield only limited gains from laser to EM pulse power.

(ii) The "lock-on" mode in which the switch closes as in the linear mode, but remains closed after termination of the laser pulse, leaving a residual voltage across the switch.

(iii) The avalanche mode in which each primary photocarrier is thought to generate multiple secondaries by impact ionization. This mode starts as a lock-on with low conductivity and the avalanche develops after a delay of approximately a nanosecond. After the avalanche starts, the closing time is also approximately one nanosecond, independent of the laser rise time. In this mode, large gains are possible $\left(>10^{3}\right)$, but the delay and slow closing time would eliminate the most interesting sub-nanosecond switching applications if they cannot be prevented. Experiments using other materials (eg. Si) and different configurations have also been done [2].

These phenomena are not well understood theoretically, but would play a basic role in the efficiency, jitter, opening behavior and lifetime of semiconductor photoswitches. These issues are likely to determine the feasibility of these photoswitches for high-power applications.

The objectives of this project were two-fold: (i) to gain an understanding of the various phenomena which occur in laser-triggered solid-state photoswitches at high fields and (ii) to bring our expertise in advanced plasma simulation to bear on modeling of semiconductor devices, which have broad industrial applications. 


\section{MILESTONES \& PROGRESS TO DATE}

- Assembled a one demensional, time-dependent Monte Carlo solid state simulation code.

- Elucidated the time step stability constraint effective in Monte Carlo device simulation.

- Implemented an implicit field algorithm that successfully relaxes the time step constraint for stability of Monte Carlo device simulation.

- Analyzed photoswitches in the linear mode, with applications to LLNL experiments, showing the gain limitation of this mode.

- Calculated photoioinization cross-sections and trap-to-band impact ionization cross-sections using the quantum-defect method.

- Using our Monte Carlo simulation code, calculated multi-phonon recombination times for selfconsistently heated electron distributions in high fields and electron mobility at high impurity concentrations.

\section{RESULTS}

\section{MONTE CARLO SIMULATION CODE}

The most widely used method for computer simulation of semiconductor devices is the driftdiffusion model. In this model, continuity equations are solved for each carrier species, with velocities proportional to the electric field, which is solved self-consistently using Poisson's equation. This method is efficient and allows the simulation of large devices, but it assumes Maxwellian distributions for the carriers. It ignores both transient carrier velocities and hot carrier effects, and requires transport coefficients (such as mobility and ionization rates) determined experimentally or by other computational methods.

Monte Carlo methods and self-consistent particle simulations, which follow a large number of individual particles have come into use in recent years. Carriers, represented as wave packets, are followed in $\mathbf{r} \mathbf{k}$ space as particles in a plasma, but are different from plasma particles in the tensor character of their mass and in the greater importance of scattering. These methods are more computer intensive than the drift-diffusion models but give a detailed representation of the carrier distribution functions. Particle simulations have successfully predicted the performance of sub-micron field-effecttransistors, which are characterized by high fieids $(\approx 100 \mathrm{kV} / \mathrm{cm})$ and short transit times, during which carrier velocities do not reach steady state.

We have developed a one dimensional time-dependent Monte Carlo code for simulation of solid state devices. This code uses a multiple-valley representation of the band structure in the non-parabolic and non-spherical approximation, and includes the most relevant scattering processes. The electric field is evaluated from the Poisson equation to perform self-consistent simulations in one dimension. It also includes a drift-diffusion model of charge carriers to allow hybrid simulations with both particles and fluids. The particles are advanced using the variable free-flight time alogirthm, using the explicit method described by Hockney and Eastwood [3] or a time-implicit algorithm which allows larger time steps for the high carrier concentrations expected in the avalanche mode. The electric field and potential are solved for on a regular one dimensional grid. Particle density is interpolated to the grid using linear weighting. The field solve can be periodic with a spatially uniform applied field, or with applied potential across the system.

Particles are organized into multiple numerical species, representing multiple bands and band minima for both holes and conduction electrons. For example, conduction electrons in GaAs might be described by one species representing the central spherical non-parabolic $\Gamma$-valley, and a second species representing the three (identicle) higher lying spherical parabolic $\mathrm{X}$-valley minima. Each numerical species can be assigned appropriate scattering processes, allowing complete flexibility to include 
additonal processes as necessary. Scattering processes currently implemented include intra-valley elastic acoustic phonon scattering, intravalley polar optical phonon emmisson and absorption, equivalent and non-equivalent intervalley acoustic phonon scattering in the deformation potential approximation, and ionized impurity scattering in the Brooks-Herring approximation. Band-to-band ionization by electron impact is modeled by the Keldysh formulation [4]. Self-scattering is included in connection with the variable time step method for choosing the particle free-flights, and is allowed to be piece-wise constant in energy.

This code [5] has been benchmarked against published results for both Silicon and GaAs. These benchmarks include calculations of steady state drift velocity versus applied electric field in homogeneous GaAs and Si., as well as inhomogeneous simulations of a submicron GaAs diode with highly doped $n^{+}$ layers employed as cathode and anode.

\section{1 (a) Time step stability}

One important constraint on self consistent simulations of both solid state devices and plasmas is numerical stability of plasma waves. This limitation imposes a maximum on the allowed time step interval between Poisson field solutions $\Delta t$, relative to the plasma frequency $\omega_{p}$, and is particularly important for simulations of devices with high carrier concentrations, such as found in heavily doped contact regions. Motivated by analysis of numerical schemes for plasma simulation, many authors have quoted the stability limit $\omega_{p} \Delta t<2$ [3]. This limit of $\omega_{p} \Delta t<2$, however, is specific to the leapfrog particle advance used in plasma simulation and is generally not applicable to algorithms used for solid state device simulation. In contrast to the leapfrog algorithm which is centered and advances the particles with a fixed time increment equal to the time step between field solves, $\Delta t$, solid state simulations typically use non-centered algorithms with a particle time step $\delta t \neq \Delta t$. Furthermore the particle time step is often picked stochastically based on mean free collision times determined not only by physical parameters but also by details of the numerical implementation such as self-scattering.

The numerical stability of typical algorithms used for Monte Carlo device simulation has been investigated, and our results are presented in detail in Ref. [6]. This analysis is applied to a variety of algorithms in different regimes; here we confine our discussion to the case of $\delta t<<\Delta t$ which is generally applicable to any scheme which only uses the electric field at the old time level in advancing the particles. This situation could correspond to the case of high collision rate (perhaps due to a large self scatter rate), or simply an attempt to ensure very accurate particle orbits. In this limit the particle advance approximates an exact orbit. Then the numerical solution corresponds to solving the Boltzmann equation exactly between times $t^{n}$ and $t^{n+1}=t^{n}+\Delta t$ with the time independent electric field, $E\left(t^{n}\right)$. A dispersion relation is obtained, which shows the surprising result that in the absence of collisions, instability occurs for all time steps. In practice, collisions allow stable simulation for finite time step. The appropriate collision rate is the rate of momentum transfer $v_{c}$, defined by the first velocity moment over the Boltzmann collision operator, and may be related to the mobility by $v_{c}=e / \mu^{*}$ with $e$ and $m^{*}$ the electron charge and effective mass. The amount of collisionality required to offset the tendancy for growth is determined by the threshold for stability (zero growth, $\gamma=0$ ),

$$
\begin{aligned}
\left(\frac{v_{c}}{\omega_{p}}\right)_{\gamma=0} & =\left(\frac{1-e^{-\alpha}-\alpha e^{-\alpha}}{1-e^{-\alpha}}\right)^{1 / 2} & , \text { for } \alpha \equiv v_{c} \Delta t \leq \alpha_{c} \\
& =\left(\frac{\alpha}{2}-\frac{1-e^{-\alpha}}{1+e^{-\alpha}}\right)^{1 / 2} & , \text { for } \alpha \equiv v_{c} \Delta t \geq \alpha_{c}
\end{aligned}
$$

and plotted in Fig. 1. Stable solutions lie above the stability threshold shown as a solid line in Fig. 1. For values of the collision rate below this threshold, unstable growth is present. $\alpha_{c}=3.72$ corresponds to the point $C$ in Fig. 1 where the threshold crosses the boundary between complex and real roots denoted by a dotted line. An approximate expression for the stability limit, valid for $\gamma \Delta t<<1$ and $v_{c} \Delta t<<1$, is given by, 


$$
\omega_{p} \Delta t \leq 2 v_{c} / \omega_{p}
$$

This condition is plotted as a straight dashed line in Fig. 1 and comparison with the exact threshold (solid line) shows that it remains a good approximation out to values of $\omega_{\mathrm{p}} \Delta t$ approaching unity.

A number of simulations have been performed to explore the stability boundary in the space of $v_{C} / \omega_{p}$ vs. $\omega_{p} \Delta t$ shown in solid line in Fig. 1 . The code used allows multiple nonparabolic, elliptic bands and scattering processes appropriate for simulation of $\mathrm{GaAs}$ or $\mathrm{Si}$. The particle advance is performed as described by Hockney and Eastwood with $\delta$ t picked randomly based upon the total scattering rate $\Gamma$ which includes self scattering. A grid with uniform spacing $\Delta x$ is used; interpolation from the particles to the grid uses standard linear weighting, and the Poisson equation is solved directly without spatial smoothing. Results from simple simulations which closely conform to the analysis are shown in Fig. 1. For these runs a single spherical parabolic band is used, and collisions correspond to elastic, isotropic scattering which is independent of energy. Simulation results are plotted as solid markers if unstable growth is observed, and as open markers if the run was observed to be stable $(\gamma \Delta t<0.01)$. The circles are from simulations with $\Gamma=v_{c}$, while triangles represent simulations with $\Gamma=5 v_{c}$. It can be seen that the stability condition implied by the boundary between solid and open markers is in reasonable agreement with the analysis, but indicates a slightly more stringent stability condition; this is due to the effects of finite temperature which are neglected in the analysis.

As an example relevant to realistic device simulations, consider $\mathrm{GaAs}$ at a doping density of $N_{D}=1.0 \times 10^{17} \mathrm{~cm}^{-3}$; assuming the electron density is equal to the doping density we have $\omega_{p} \approx 2.0 \times 10^{13} \mathrm{~s}^{-1}$. At a lattice temperature of $T=300 \mathrm{~K}$, the low field mobility is $\mu=5.3 \times 10^{3} \mathrm{~cm}^{2} / \mathrm{V} \cdot \mathrm{s}$; this corresponds to an effective collision frequency $v_{c}=5.0 \times 10^{12} \mathrm{~s}^{-1}$. Then $v_{c} / \omega_{p} \approx 0.25$, and the stable time step limit is predicted to be $\omega_{p} \Delta t \approx 0.5$ or $\Delta t=2.6 \times 10^{-14} \mathrm{~s}$. At a lattice temperature $T=77 \mathrm{~K}$, $\mu=9.2 \times 10^{3} \mathrm{~cm}^{2} / \mathrm{V} \cdot \mathrm{s}$ corresponding to $v_{c}=2.9 \times 10^{12} \mathrm{~s}^{-1}$. Then $v_{c} / \omega_{p} \approx 0.15$ and the stability limit is $\omega_{p} \Delta t=0.3\left(\Delta t=1.5 \times 10^{-14} \mathrm{~s}\right)$. Simulations of the two cases described above $\left(T=300 \mathrm{~K}, \omega_{p} \Delta t=0.5 ; T=77 \mathrm{~K}\right.$, $\left.\omega_{p} \Delta=0.3\right)$ performed using realistic models for GaAs show weak instability; stability requires somewhat smaller values of the time step consistent with the effect of finite pressure. Unstable runs were observed to saturate by heating the electrons. In some cases, mobilities were noticably reduced and significant numbers of electrons promoted to the upper valleys.

\section{1 (b) Large time step algorithm}

Frequent solution of the Poisson equation to resolve plasma oscillations can be a sizable computational burden. Caution suggests that the condition presented above not be approached to closely, since finite pressure effects slightly lower the stability limit. Additionally, near the stability limit unphysical heating of the carriers may be a more insidious effect than the catastrophic instability which occurs well above the limit. The necessity of using advanced time levels for numerical stability with $\omega_{p} \Delta \gg>1$ has long been known in the case of collisioniess plasma simulation, and stable large time step simulations have been achieved using time-implicit methods [7].

The key ingredient for large time step stability is to advance the particles using the advanced electric field $E^{n+1}$, such as

$$
x^{n+1}=x_{0}+\beta \Delta t^{2} \frac{q E^{n+1}}{m^{*}}
$$

where $x_{0}$ depends only on quantities at the past time level $\boldsymbol{t}^{n}$ and $\beta$ is the implicitness parameter. Because the new field depends on the new particle positions through the solution to the Poisson equation, however, an implicit solution for the electric field is required. The implicit field equation may be found by writing the Poisson equation at the new time level, and linearizing the charge density with respect to perturbations due to the advanced field,

$$
\frac{\partial E^{n+1}}{\partial x}=\frac{4 \pi}{\varepsilon} \rho^{n+1} \equiv \frac{4 \pi}{\varepsilon}\left\{\rho_{0}\left(x_{0}\right)+\delta \rho\right\}
$$


The perturbation to the charge density, $\delta \rho$, may be expressed in terms of the perturbation to the particle position $\delta x$ by,

$$
\delta \rho=-\frac{\partial}{\partial x}\left(\rho_{0} \delta x\right)=-\frac{\partial}{\partial x}\left(\rho_{0} \frac{q}{m^{*}} \beta \Delta t^{2} E^{n+1}\right) .
$$

Substituting into Eq. (1.4), and rearranging, the field equation becomes

$$
\frac{\partial}{\partial x}\left\{(1+\chi) \frac{\partial \phi}{\partial x}\right\}=-\frac{4 \pi}{\varepsilon} \rho_{0} \quad, \quad \chi \equiv \frac{4 \pi}{\varepsilon} \rho_{0} \frac{q}{m^{*}} \beta \Delta t^{2}=\beta\left(\omega_{p} \Delta t\right)^{2},
$$

with $\chi$ the effective susceptibility due to the partial advance of the particles to $x_{0}$. Strict implementation of such a scheme requires writing these equations with the spatial derivatives replaced by finite differences generalized to include the interpolation between the grid and particles. This leads to a matrix system for the new electric field which is completely consistent with the particle push but has a larger stencil than the original explicit system. Simplified differencing (and reduced computational stencil) can be obtained by simply writing Eq. (1.6) in finite difference form; this is appropriate if $\omega_{p} \Delta t$ is not too large.

We have adapted these implicit plasma techniques to semiconductor modeling and demonstrated stable simulation for $\omega_{p} \Delta t$ larger than the limit given in Section 1(a). At each time step, particles which undergo one or more collisions $(\delta t<\Delta t)$ are advanced explicitly. These collisional particles contribute only to $\rho_{0}$ and not to the susceptibilities. Particles which do not undergo a collision $(\delta \geq \Delta r)$ are advanced implicitly by performing a partial push and accumulating the necessary susceptibilities. Then the implicit field equation is solved and the positions of the implicit particles corrected, completing the time step. Calculations of a submicron GaAs diode [8] have been performed as a realistic test. The diode is composed of a $0.25 \mu \mathrm{m}$ undoped active layer between $0.35 \mu \mathrm{m} n^{+}$-layers doped at density $N_{D}=2.0 \times 10^{17}$ $\mathrm{cm}^{-3}\left(\omega_{p} \approx 2.7 \times 10^{13} \mathrm{~s}^{-1}\right)$. The simulation model is substantially the same as used by Tomizawa et al., and for their time step, $\Delta t=1.0 \times 10^{-14} \mathrm{~s}$, similar results are obtained. Figure 2 shows time histories of the average particle energy and electrostatic energy from simulations with an applied voltage of 0.25 volts (rising from zero in $1.0 \mathrm{ps}$ ) and lattice temperature of $77 \mathrm{~K}$. An explicit calculation with $\Delta t=1.5 \times 10^{-14} \mathrm{~s}$ is unstable (as predicted above for $\omega_{p} \Delta t \approx 0.42$ ), while in contrast, the implicit calculation $(\beta=0.75)$ with $\Delta t=8.0 \times 10^{-14} \mathrm{~s}\left(\omega_{\mathrm{p}} \Delta t=2.24\right)$ is well behaved. The increase in time step which can be realized is limited, because as $\Delta t$ is increased, the fraction of particles which are treated implicitly decreases. As $\Gamma \Delta t$ becomes of order unity, most of the particles are treated explicitly, and the stability limit of Eq. (1.1) becomes effective. Although the time savings in one dimension is modest, appreciable gains in multi-dimensional simulation might be realized because of the increased computational burden of the Poisson solve.

\section{PHOTOSWITCHES: ANALYSIS OF LINEAR MODE}

In the transmission line experiments of Ref. 1, a GaAs slab of thickness $d$ is inserted at the center of a parallel plate transmission line as shown in Fig. 3. The line is initially charged to a bias electric field $E_{0}$ and a laser pulse with energy $W_{\text {las }}$ is applied, causing the GaAs slab to become conducting. A current of density $J$ flows and two transverse electromagnetic (TEM) pulses with electric field $E$ and magnetic field $B$ propagate outward, so that the residual electric field on the GaAs slab is $E_{s} \equiv E_{0}-E$. From Ampere's law, using symmetry,

$$
B=\frac{2 \pi}{c} J d
$$

Gaussian cgs units are used here, $c$ denotes the speed of light, for TEM propagation $E=B$, and edge effects are neglected. The current density is

$$
J=e n_{e} u_{d}
$$

where $e$ is the magnitude of the electron charge, $n_{e}$ is the conduction electron density and $u_{d}$ is the drift velocity. If the laser rise time allows conduction electrons to reach steady-state, $u_{d}$ is a function of the residual field, $E_{0}-E$ applied to the GaAs slab and is shown in Fig. 4. From Eqs. (2.1) and (2.2), 


$$
E=\frac{2 \pi e}{c} d n_{e} u_{d}
$$

This may be rearranged to define the load line shown in Fig. 4,

$$
E_{0}-E=E_{0}-\frac{2 \pi e}{c} d n_{e} u_{d}
$$

and its intersection with the drift velocity determines the operating point. Under dark conditions, the load line is vertical, with $n_{e}=0$ and $E=0$. As laser light generates conduction electrons, $n_{e}$ increases and the load line sweeps to the left. The photoswitch may operate either in the "saturated" regime where the load line remains above the tangent $A$ in Fig. 4, or in the "unsaturated" regime where it reaches below tangent $B$. Between tangents A and B, lies the Gunn domain regime and we assume here that the laser rise time is sufficiently fast to prevent significant instability when the photoswitch passes through this regime. The saturated regime occurs only for bias fields exceeding $\approx 14 \mathrm{kV} / \mathrm{cm}$ and the electron densities corresponding to tangents $A$ and $B$ are found from Fig. $4, n_{A}=c E_{0} /\left(2 \pi e d u_{A}\right)$, with a similar expression for $n_{\mathrm{B}}$. For example, $E_{0}=24 \mathrm{kV} / \mathrm{cm}$ and $d=0.1 \mathrm{~cm}$ give $n_{\mathrm{A}}=3.5 \times 10^{14} \mathrm{~cm}^{-3}$ and $n_{\mathrm{B}}=4.5 \times 10^{14} \mathrm{~cm}^{-3}$.

In the saturated regime, $n_{e}<n_{A}$, the drift velocity is approximately constant and the pulse electric field is given by Eq. (2.3) with $u_{d}=u_{\infty} \approx 10^{7} \mathrm{~cm} / \mathrm{s}$. In the unsaturated regime, $n_{e}>n_{\mathrm{B}}$, the drift velocity is $u_{d}=\mu\left(E_{0}-E\right)$, where $\mu=2.6 \times 10^{6} \mathrm{~cm}^{2} / \mathrm{s} \cdot \mathrm{stV}$ for intrinsic GaAs. In this case, Eq. (2.3) gives

$$
E=\frac{E_{0}}{1+n^{*} / n_{e}}, \quad n^{*}=\frac{c}{2 \pi e \mu d}
$$

but in the unsaturated regime, the second term in the denominator is small and we have neariy total switching, i.e. , $E \approx E_{0}$.

In the linear mode, each photon absorbed in the photoconducting material generates a conduction electron and the recombination time, $\tau_{r e c}$, is constant, whence

$$
\frac{d n_{e}}{d t}=\frac{I(t)}{h v d}-\frac{n_{e}}{\tau_{r e c}}
$$

Here, $h v$ is the photon energy and $I(t)=I_{0} g(t)$ is the absorbed laser intensity. The laser pulse shape is chosen as $g(t)=\sin ^{2}\left(\pi t / 2 \tau_{\text {las }}\right)$ which has a full width at half maximum (FWHM) equal to $\tau_{\text {las. }}$ Solving Eq. (2.6) gives the electron density as a function of time which is in the form of a pulse having a maximum, $n_{\max }$, and a width, $\tau$, given in Table 1 for two typical values of the ratio $\tau_{\text {lad }} \tau_{\text {rec }}$. The normalization density in the table is $n_{0}=I_{0} \tau_{\text {red }} / h v d$.

We may apply this analysis to the experimental results presented in Fig. 9 of Ref. 1. First consider the data of Fig. 9(b), which may be replotted as $E / E_{0}$ vs. $E_{0}$ as shown in Fig. 5(a). At high switch field, the output field appears to saturate at approximately $4 \mathrm{kV} / \mathrm{cm}$. This my be used in Eq. (2.3) to obtain the electron density, $n_{e}=1.3 \times 10^{14} \mathrm{~cm}^{-3}\left(d=0.1 \mathrm{~cm}\right.$, and assuming a saturated drift velocity of $u_{\infty}=1 \times 10^{7}$ $\mathrm{cm} / \mathrm{s})$. This electron density may then be used to estimate the low field mobility from the slope of $E / E_{0}$, to obtain $\mu=1 \times 10^{3} \mathrm{~cm}^{2} \mathrm{~N}$.s. This value of the mobility is nearly a factor of ten smaller than the value for intrinsic GaAs, consistent with the fact that this data was obtained with neutron irradiated material (to decrease the recombination time). One can then confirm that the data presented in Fig. 9(a) of Ref. 1 for $E / E_{0}$ as a function of laser energy (for $E_{0}=4.8 \mathrm{kV} / \mathrm{cm}$ ) is in the unsaturated regime; hence Eq. (2.5) should apply. Using the just calculated mobility, one obtains $n^{*}=3.3 \times 10^{14} \mathrm{~cm}^{-3}$. With electron density proportional to laser energy, and using our previously calculated value of the electron density, $n_{e}=1.3 \times 10^{14} \mathrm{~cm}^{-3}$ for a laser energy of $72 \mu \mathrm{J} / \mathrm{cm}^{2}$, Eq. (2.5) can be plotted with the data of Pocha and Druce as shown in Fig. 5(b). The recombination time may also be estimated. With the estimate of Ref. 1 for the fractional absorbed laser energy as $10 \%$, we estimate notlaser $/ \tau_{\text {rec }}=I_{0} \tau_{\text {laser }} / \mathrm{hvd}=3.6 \times 10^{14} \mathrm{~cm}^{-3}$, and hence $R \approx 0.36$. Thus from Table $1, \tau_{\text {rec }}=0.5 \tau_{\text {laser }}=50 \mathrm{ps,} \mathrm{and} \mathrm{the} \mathrm{output} \mathrm{pulse} \mathrm{should} \mathrm{be} \mathrm{just}$ somewhat longer than the laser pulse, consistent with Fig. 6(a) of Ref. 1.

The electromagnetic energy in each pulse is obtained from Poynting's vector, $W_{e m} \approx A E^{2} c \tau / 4 \pi$, where $A$ is the switch area. Here time integration of $E^{2}$ has been estimated by multiplying by $\tau$. The gain, 
relative to the absorbed laser energy, is $G=W_{e m} / W_{\text {las }}$, where $W_{\text {las }}=A I_{0} \tau_{\text {las }}$. Substituting the expressions for $E$ corresponding to the saturated and unsaturated regimes, yields the estimates

and

$$
G_{s a t}=\frac{\pi e^{2}}{c} \frac{I_{0} u_{\infty}^{2} \tau_{\text {rec }}^{2}}{(h v)^{2}}\left(\frac{n_{e}}{n_{0}}\right)^{2} \frac{\tau}{\tau_{\text {las }}}
$$

$$
G_{\text {unsat }}=\frac{c E_{0}^{2}}{4 \pi I_{0}} \frac{\tau}{\tau_{\text {las }}}
$$

For the experiments of Ref. $1, d=0.1 \mathrm{~cm}$, and for the linear mode (Fig. 6 of Ref. 1), $E_{0}=24 \mathrm{kV} / \mathrm{cm}=80$ $\mathrm{stV} / \mathrm{cm}$. This corresponds to a voltage of $12 \mathrm{kV}$ over the $0.5 \mathrm{~cm} \mathrm{GaAs} \mathrm{slab.} \mathrm{The} \mathrm{laser} \mathrm{energy} \mathrm{incident} \mathrm{on}$ the slab $0.5 \mathrm{~cm} \times 2.0 \mathrm{~cm}$ was $1.32 \mathrm{~mJ}$ with a pulse length of $100 \mathrm{ps}$. This gives an incident intensity of $1.32 \times 10^{14} \mathrm{erg} / \mathrm{cm}^{2} . \mathrm{s}$. The absorption length of the $1.06 \mu \mathrm{m}$ light in the $\mathrm{GaAs}$ samples had been measured to be $\approx 1 \mathrm{~cm}$, giving an absorbed intensity $I_{0}=1.32 \times 10^{13} \mathrm{erg} / \mathrm{cm}^{2} . \mathrm{s}$. Assuming a recombination time $\tau_{\text {rec }}=100 \mathrm{ps}$, this case gives $n_{0}=7 \times 10^{15} \mathrm{~cm}^{-3}$, and from Table 1 with $\tau_{\text {las }} / \tau_{\text {rec }}=1$, the maximum value of $n_{e}$ is $0.54 \times 7 \times 10^{15} \mathrm{~cm}^{-3}=3.8 \times 10^{15} \mathrm{~cm}^{-3}$. This is above the value $n_{\mathrm{B}}=4.5 \times 10^{14} \mathrm{~cm}^{-3}$ computed earier, putting this experiment in the unsaturated regime and the full voltage should be switched $\left(E \approx E_{0}\right)$. Note that this is indeed observed in Fig. 6 of Ref. 1. The gain in this case is given by Eq. (2.7), G=1.2.

We may consider if the gain could have been larger with a lower intensity for which the photoswitch would operate in the saturated regime. This requires a maximum electron density smaller than $n_{\mathrm{A}}=3.5 \times 10^{14} \mathrm{~cm}^{-3}$, or from Table $1, n_{0}<n_{\mathrm{A}} / 0.54=6.5 \times 10^{14} \mathrm{~cm}^{-3}$ and from the definition of the normalization density, an intensity $I_{0}<h v d n_{0} / \tau_{\text {rec }}=1.2 \times 10^{12} \mathrm{erg} / \mathrm{cm}^{2} . \mathrm{s}$. Operating at this intensity to achieve the maximum gain, Eq. (2.7) yields $G=3.6$. The switched field in this case, given by Eq. (2.3) with $u_{d}=u_{\infty}=10^{7} \mathrm{~cm} / \mathrm{s}$, is $E=35.2 \mathrm{stV} / \mathrm{cm}$ or $10 \mathrm{kV} / \mathrm{cm}$, which is only a fraction of the applied field of $24 \mathrm{kV} / \mathrm{cm}$. Raising the applied field to increase the gain is not possible in the present case because the switch has been found experimentally to go to the lock-on mode for $E_{0}>24 \mathrm{kV} / \mathrm{cm}$.

\section{TREATMENT OF DEEP-LEVEL IMPURITIES (TRAPS)}

An impurity can either introduce an additional valence electron (donor) or can lack a valence electron (acceptor) relative to the perfect crystal. In the case of a donor, the additional electron is in the conduction band, but it can either be free or trapped in orbit around the positive center of the donor ion. Similarly, for an acceptor the resulting hole in the valence band can also be free or trapped by the negative center of the acceptor ion. The electronic states of such impurities are treated as hydrogen atoms by replacing the electron mass by the equivalent mass $m^{*}$ of either the conduction electron or hole, and taking into account the dielectric constant, $K$, of the crystal by replacing $e^{2}$ by $e^{2} / K$ in the orbiting electron wave functions and in the formulas expressing the hydrogenic excitation and ionization energies. In GaAs, for example, $m^{*}=0.067 m_{e}$ and $K=12.5$, where $m_{e}$ is the electron mass. A singly ionized donor has an ionization energy $E_{d}=E_{\mathrm{H}}\left(m^{*} / m_{\mathrm{e}} K^{2}\right)$, where $E_{\mathrm{H}}=13.6 \mathrm{eV}$ is the hydrogen ionization energy. Thus the energy level of the donor is $5.8 \mathrm{meV}$ below the conduction band minimum and its excited states form a hydrogenic series of energy states, $E_{\mathrm{n}}=E_{\mathrm{d}} / n^{2}$, located between the "ground" state $E_{d}$ and the conduction band minimum. Because of these small energies, compared to the band gap of $1.4 \mathrm{eV}$, such impurities are called "shallow" impurities. The orbit radius is $a^{*}=a_{0}\left(m_{e} K / m^{*}\right)$, where $a_{0}=0.53 \AA$ is the Bohr radius. Thus the orbit has a radius close to $100 \AA$ encompassing many crystal cells and the Fourier transform of the electron wave function occupies a small extent in the Brillouin zone. The configuration of such a shallow donor is represented schematically in Fig. 6(a). Similar results are found for acceptor levels above the valence band, and doping with such shallow impurities is commonly used to provide charge carriers in semiconductors.

However, semiconductors also have impurities or flaws in their crystals which do not conform with this hydrogenic model. These impurities are also donors or acceptors, but 
(i) Their energy level lies in mid-gap, much further from the conduction or valence bands than can be explained by the hydrogenic model;

(ii) Their properties vary and do not depend only on the host crystal;

(iii) They generally do not have bound excited states.

Such "deep-level" impurities (or flaws) are also refered to as "traps" and they play an important role in the photoconductive properties of semiconductors.

In the hydrogenic model, the impurity is assumed to have a Coulomb potential over the entire range of distances from the center, $r=(0, \infty)$. Wave functions are solutions of the Schrodinger equation, and the energy levels, $E_{n}$, are found in a well-known manner by applying boundary conditions at $r=0$. It is expected that deep-level impurities do not conform to this model because their potential near the center is more complex and stronger than a Coulomb potential. In the "quantum defect" (QD) method, this difficuity is avoided by solving for the wave functions only in the region far enough from the center to justify application of a Coulomb potential and setting the energy in the Schrodinger equation equal to the experimentally observed value, $E_{d}=-R^{*} / N^{2}$. Here, $R^{*}=E_{\mathrm{H}}\left(m^{*} / m_{\mathrm{e}} K^{2}\right)$ is the hydrogenic value of the ionization energy and $v$ is an empirical parameter chosen to match the observed energy level. This $Q D$ parameter ranges between $v=0$ and $v=1$. For large $r$, the wave functions of the $Q D$ method take on the simple form,

$$
\Psi_{v}(r)=\frac{N_{v}}{\sqrt{4 \pi}} r^{v-1} e^{-r / v a^{*}}
$$

where $N_{\mathrm{v}}=\left(2 / v a^{*}\right)^{v}\left(v a^{*}\right)^{-1 / 2} / \Gamma(v+1)$ is the normalization constant. For $v=1$, this wave function reduces to the hydrogenic result and in the limit $v \rightarrow 0$, it reproduces the result of a model of deep-level impurities which assumes a potential in the form of a delta function [9].The effect of deep-level impurities (traps) on carrier emission and capture is presented. Photoionization of the impurities and trap-to-band impact ionization cross-sections are modeled using the quantum-defect method [10] Non-radiative emission and capture are represented using the multiphonon cross-sections and activation energies for impurities (or defects) which have been characterized experimentally [11].

\section{3 (a) Photoionization of deep-level impurities}

The QD method has been applied to photoionization of a deep-level impurity as represented schematically in Fig. $6(\mathrm{~b})$. The photon cross-section for this process is evaluated using the Bom approximation with the function $\Psi_{v}$ for the initial electron state and a plane wave as final state. This computation yields

$$
\sigma_{p h}=\frac{2 \pi \alpha_{0}\left(a^{*}\right)^{2}}{3 n} \frac{v^{2} 2^{2} f(y)}{\sqrt{y}(1+y)^{v}}
$$

where

$$
f(y)=\left\{\frac{\sin [(v+1) \alpha]}{\sqrt{y}}-\frac{(v+1) \cos [(v+2) \alpha]}{\sqrt{1+y}}\right\}^{2}
$$

$y=\left(h v / E_{d}\right)-1, \alpha=\arctan \left(y^{1 / 2}\right), n$ is the index of refraction of the crystal and $\alpha_{0}=1 / 137$ is the fine structure constant. These formulas may be applied to a mid-gap impurity in $\mathrm{GaAs}$ with $E_{d}=0.78 \mathrm{eV}$. The hydrogenic ionization energy $R^{*}=5.8 \times 10^{-3} \mathrm{eV}$, calculated earlier, gives $v=\left(R^{*} / E_{d}\right)^{1 / 2}=0.086$ and light at $1.06 \mu \mathrm{m}$ for which $h v=1.17 \mathrm{eV}$ gives $y=0.5$. Thus $f(y)=0.4$ and taking the index of refraction as $n=K^{1 / 2}$, where $K=12.5$, the photoionization cross-section is $\sigma_{p h}=1.8 \times 10^{-17} \mathrm{~cm}^{2}$. The resulting absorption length is $l_{a b s}=\left(n_{t,} \sigma_{p h}\right)^{-1}$, where $n_{t r}$ is the density of occupied deep-level impurities. For example, taking $n_{t r}=10^{17}$ $\mathrm{cm}^{-3}$ gives $l_{a b s}=0.55 \mathrm{~cm}$. 
3 (b) Impact ionization of deep-level impurities

The QD method may also be applied to evaluate the electron impact ionization of a deep-level impurity as shown schematically in Fig. 6(c). Such a process could be expected to play a role in the avalanche mode. For an incident electrcn with energy $E_{\mathrm{i}}$ above the bottom of the conduction band this transition is also evaluated using the Born approximation. A plane wave is used as initial state of the incident electron and the function $\Psi_{v}$ as initial state for the electron trapped around the impurity center. The final states of both the scattered and emitted electrons are plane waves and significant simplifications are obtained if $E_{i}$ is assumed close above the theshold $E_{d}$. The incident electron cross-section is

$$
\sigma_{i}=2\left(a^{*}\right)^{2} 2^{v} v^{4}\left[\frac{E_{i}}{E_{d}}-1\right]^{2} \sin ^{2}[(v+1) / 4]
$$

and the ionization time is $\tau_{i}=\left(\sigma_{i} v_{i} n_{t r}\right)^{-1}$, where $v_{e}=\left(2 E_{i} / m^{*}\right)^{1 / 2}$ is the incident electron velocity. For occupied mid-gap impurities in GaAs at density $n_{t r}=10^{17} \mathrm{~cm}^{-3}$ considered earlier and for an incident electron with energy $E_{i}=2 E_{d}$, the ionization time is $\tau_{i}=6 \mathrm{~ns}$. This is a long time compared to the photoswitch operation.

\section{3 (c) Multi-phonon recombination}

The analysis of Section 2 has shown that carrier recombination plays an important role in the reopening of photoswitches. This recombination occurs by capture of conduction electrons into deep-level impurities. Radiative capture with emission of a photon and Auger recombination, which is the reverse of electron impact ionization, can be shown to be negligible. The most important capture process appears to be multiphonon capture which involves interaction berween the electron states and lattice vibrations of the crystal [12].

Multiphonon emission and capture considers a well-localized impurity center with an electron orbit not much larger than the distance between nearest lattice points. In this case, the equilibrium positions of the lattice ions in the vicinity of the impurity depend on whether the impurity is neutralized by an orbiting electron or un-neutralized, with its electron free in the conduction band. This electronic state of the impurity affects most strongly the radial or "breathing" motion of the surrounding lattice ions. The positions of these surrounding ions may be represented conceptually by a single variable, $X$, and their motion in the breathing mode may be considered as a harmonic oscillator having a parabolic potential vs. $X$. It is convenient to express the variable $X$ in terms of the dimensionless "configuration coordinate" $Q=2 \pi\left(v_{i} M / h\right)^{1 / 2 X}$, where $v_{i}$ is the oscillator frequency and $M$ is its equivalent mass.

We may now draw a configuration coordinate (CC) diagram, as shown in Fig. 7 , in which the sum of the elastic and electronic potential energies is drawn as a function of $Q$. Two parabolic energy curves have been drawn in Fig. 7, the lower-left curve corresponds to the state where the electron is in orbit (neutralized impurity center) and the upper-right curve corresponds to the state where the electron is free (un-neutralized center). Note that the equilibrium position, located at the potential minimum, changes from $Q_{1}$ to $Q_{2}$ as the center becomes ionized. The height of the potential minimum also changes by an amount equal to the ionization energy, $E_{d}$, of the impurity center. The $\mathrm{CC}$ diagram also shows the FranckCondon energy, $E_{F C}$, defined as the elastic energy corresponding to a lattice deformation between the potential minima at $Q_{1}$ and $Q_{2}$. This energy is often written as $E_{F C}=S V_{i} h$, where $S$ is called the HuangRhys factor. This diagram is useful to interpret phonon-assisted optical transitions. In such transitions, a photon causes ionization of the impurity center from A to B where the lattice is no longer in an equilibrium condition. Lattice vibrations with phonon emission bring the system to point $C$. The transition $C D$ occurs when an electron is captured with photon emission, and lattice vibrations with phonon emission bring the system back to point $A$. In this series of transitions the photon emitted as fluorescence along $\mathrm{CD}$ has a lower frequency than the photon absorbed along $\mathrm{AB}$. The difference between absorption and fluorescence frequencies is $2 E_{F C}$.

When the energy levels intersect as shown in Fig. 7, non-radiative transitions can occur at the crossing point. The energy $E_{B}$ is the minimum vibration energy required to allow an electron to make a 
transition from free to trapped state. Thus $E_{B}$ represent a vibrational capture barrier. The geometry of Fig. 7 allows $E_{B}$ to be expressed as

$$
E_{B}=\frac{\left(E_{d}-E_{F C}\right)^{2}}{4 E_{F C}}
$$

and for a thermal distribution of vibrational energies at temperature $T$, the capture cross-section is

$$
\sigma_{c}=\sigma_{\infty} \exp \left(-E_{B} / T\right)
$$

where $\sigma_{\infty}$ may be expressed in terms of the elastic properties of the crystal and $k_{B}$ is the Boltzmann constant. Non-radiative emission, which can also occur when the vibrational energy exceeds $E_{d}+E_{B}$ is related to capture by detailed balance and the emission rate is

$$
e_{n r}=\gamma_{n} T^{2} \sigma_{\infty} \exp \left[-\frac{E_{d}+E_{B}}{T}\right]
$$

where $\gamma_{n}=4 \pi(6 \pi)^{1 / 2} m^{*} k_{B}{ }^{2} / h^{3}$.

Numerous non-radiative emission and capture experiments have been done and plots of $\sigma_{\infty}$ and $T^{2} / e_{n r}$ vs. $1 / T$ display straight-line segments as predicted by the preceeding equations. These plots allow the determination of $\sigma_{\infty}$ and $E_{B}$ (often called the activation energy) for a variety of GaAs samples. Typical values are $\sigma_{\infty}=10^{-15}$ to $10^{-14} \mathrm{~cm}^{-2}$ and $E_{B}=0.1$ to $0.4 \mathrm{eV}$. For example, $\sigma_{\infty}=5 \times 10^{-15} \mathrm{~cm}^{2}$ and $E_{B}=0.1 \mathrm{eV}$ give a capture cross-section $\sigma_{c}=10^{-16} \mathrm{~cm}^{2}$ at $300 \mathrm{~K}$. For an empty trap density $n_{t r}=10^{17} \mathrm{~cm}^{-3}$, the recombination time of an electron at $0.026 \mathrm{eV}(300 \mathrm{~K})$ is $\tau_{r e c}=\left(\sigma_{\mathrm{c}} n_{t r} v_{e}\right)^{-1}=2.7 \mathrm{~ns}$. Note that the ionization and recombination times depend on the energy level, activation energy, and densities of occupied and empty traps, which vary widely for various samples.

In high-field applications of photoswitches, hot electrons, having a significant energy, $E_{e}$ over the band minimum can be generated. The evaluation of the vibrational energy barrier, $E_{B}$, from Fig. 7 and the experimental data are applicable to conduction electrons near the bottom of the conduction band. For hot electrons, the height of the potential minimum in Fig. 7 becomes $E_{d}+E_{e}$ and the vibrational barrier is increased to

$$
E_{B}^{\prime}=E_{B}+\frac{1}{4 E_{F C}}\left[2\left(E_{d}-E_{F C}\right) E_{e}+E_{e}^{2}\right]
$$

where the Franck-Condon energy is found from Eq. (3.5),

$$
E_{F C}=E_{d}+2 E_{B}\left[1-\sqrt{1+E_{d} / E_{B}}\right]
$$

For an impurity with ionization energy $E_{d}=0.78 \mathrm{eV}$ and activation energy $E_{B}=0.1 \mathrm{eV}$, the Franck-Condon energy is $E_{F C}=0.39 \mathrm{eV}$. For an electron at energy $E_{e}=0.2 \mathrm{eV}$, the barrier is raised to $0.23 \mathrm{eV}$ and the recombination time would be $\approx 130 \mathrm{~ns}, 50$ times longer than for an electron at $300 \mathrm{~K}$. This strong dependence of the recombination time on the electron energy may play a role in the "lock-on" mode observed at high applied fields, since electrons in this case acquire large energies.

\section{3.(d) Monte Carlo calculations}

The analysis of Section 3(c), offers the intriguing possibilty that multi-phonon recombination rates are significantly reduced for electron distribution functions that are heated under high field conditions. We have calculated self consistent rates using heated electron distributions obtained from our Monte Carlo simulation code. These rates are calculated as

$$
\left(n_{t r} \tau_{r e c}\right)^{-1}=\int \sigma_{c}\left(E_{e}\right) \cup\left(E_{e}\right) f\left(E_{e}\right) d E_{e}
$$

where the cross section $\sigma_{\mathrm{c}}$ as a function of electron energy $E_{e}$ comes from combining Eq. (3.6) with the barrier energy, Eq. (3.8), and the velocity $v$ and distribution function $f$ as a function of electron energy are provided by Monte Carlo simulation. By performing several simulations at different field strengths, the recombination time as a function of electric field is obtained, as shown in Table 2 . The large increase in recombination time as a function of field strength is striking, and would be consistent with the long conduction times observed experimentally. This is in sharp constrast with other authors [13], who have 
argued for recombination times decreasing with field strength as a way of explaining delayed current onset.

The analysis of the previous section also indicates that the low field mobility is quite small, and that the recombination times observed in the experiments imply very large impurity concentrations. We have used our Monte Carlo simulation model to calculate low field mobilities for several values of the ionized impurity concentration; these results are shown in Table 3. It is clear that ionized impurity concentrations due to photoionizaton, $n_{l}=n_{e}=10^{14} \mathrm{~cm}^{-3}$ do not explain the very small mobility observed. If the impurities are highly compenstated, so that $n_{p}>n_{e}$ then order of magnitude reductions of the mobility are possible for $n_{\mathrm{i}} \approx 10^{18} \mathrm{~cm}^{-3}$. This value is in line with the estimates for the trap concentrations neccessary to explain the photoionization and recombination times.

\section{REFERENCES}

[1] M. D. Pocha, and R. L. Druce, "35-kV GaAs Subnanosecond Photoconductive Switches," IEEE Trans. Electron Devices, Vol. 37, pp. 2486-2492, 1990.

[2] F. J. Zutavem, G. M. Loubriel, M. W. O'Malley, L. P. Shanwald, W. D. Helgeson, D. L. McLaughlin, and B. B. McKenzie, "Photoconductive Semiconductor Switch Experiments for Pulsed Power Applications," IEEE Trans. Electron Devices, Vol. 37, pp. 2472-2477, 1990.

[3] R. W. Hockney and J. W. Eastwood, Computer Simulation Using Particles, pp. 353-408. New York: Adam Hilger, 1988.

[4] L. V. Keldysh, "Kinetic Theory of Impact Ionization in Semiconductors," Soviet Physics JETP, Vol. 37, pp. 509-518, 1960.

5] P.W. Rambo and J. Denavit, "Monte Carlo Modeling of Solid-State Photoswitches," Proceedings of SPIE 1873, 225 (1993).

[6] P. W. Rambo and J. Denavit, "Time Stability of Monte Carlo Device Simulation," IEEE Trans. on Computer-Aided Design 12, 1734 (1993).

[7] J. Denavit, "Time Filtering Particle Simulations with $\omega_{p} \Delta \triangleright>1$, , J. Comput. Phys. 42,337 (1981); A. B. Langdon, B. I. Cohen, and A. Friedman, "Direct Implicit Large Time-Step Particle Simulation of Plasmas," J. Comput. Phys. 51, 107 (1983).

[8] K. Tomizawa, Y. Awano, N. Hashizume, and M. Kawashima, "GaAs $n^{+}-i(n)-n^{+}$diode," IEE Proc. 129, 131 (1982).

[9] G. Lucovsky, "On the Photoionization of Deep Impurity Centers in Semiconductors," Solid State Comm., Vol. 3, pp. 299-302, 1965.

[10] H. B. Bebb, "Application of the Quantum-Defect Method to Optical Transitions Involving Deep Effective-Mass-Like Impurities in Semiconductors," Phys. Rev., Vol. 185, pp. 1116-1126, 1969.

[11] G. N. Martin, A. Mitonneau, and A. Mircea, "Electron Traps in Bulk and Epitaxial GaAs Crystals," Electronics Letters, Vol. 13, pp. 190-192, 1977; D. I. Desnica, "Characterization of Deep Traps in SemiInsulating Gallium Arsenide," J. Electronic Materials, Vol. 21, pp. 463-471, 1992.

[12] C. H. Henry and D. V. Lang, "Nonradiative capture and recombination by multiphonon emission in GaAs and GaP," Phys. Rev., Vol. B15, pp. 989-1016, 1977; B. K. Ridley, Quantum Processes in Semiconductors. Oxford: Oxford Press, 1988.

[13] W.T. White III, et al., "Modeling GaAs High-Voltage, Subnanosecond Photoconductive Switches in One Spatial Dimension," IEEE Trans, on Electron. Devices 37, 2532 (1990). 


\begin{tabular}{|c|c|c|c|}
\hline$\tau_{\text {rec }} \tau_{\text {las }}$ & 0.1 & 0.5 & 1.0 \\
\hline$R=n_{e} /$ no $_{0}$ & 0.98 & 0.75 & 0.54 \\
\hline$\tau \tau_{\text {Ias }}$ & 1.02 & 1.21 & 1.5 \\
\hline
\end{tabular}

Table 1: Pulse shape attributes from solution of Eq. (2.6) of text.

\begin{tabular}{|c|c|c|}
\hline$E(\mathrm{kV} / \mathrm{cm})$ & $\tau_{r e c}\left(E_{0}=0.78 \mathrm{eV}, E_{B}=0.42 \mathrm{eV}\right)$ & $\tau_{\text {rec }}\left(E_{0}=0.78 \mathrm{eV}, E_{B}=0.10 \mathrm{eV}\right)$ \\
\hline 0.0 & 1.0 & 1.0 \\
\hline 2.0 & 1.1 & 1.3 \\
\hline 4.0 & 1.8 & 2.5 \\
\hline 6.0 & 4.4 & 7.3 \\
\hline 8.0 & 8.1 & 14.1 \\
\hline 10.0 & 12.7 & 27. \\
\hline 15. & 23. & 51. \\
\hline
\end{tabular}

Table 2: Multi-phonon recombination times calculated from self-consistent Monte Cario electron distributions; recombination times $\tau_{\text {rec }}$ are normalized to zero field value.

\begin{tabular}{|c|c|c|c|}
\hline$n_{i}\left(\mathrm{~cm}^{-3}\right)$ & $\mu\left(\mathrm{cm}^{2} / \mathrm{V} \cdot \mathrm{s}\right): n_{e}=n_{i}$ & $\mu\left(\mathrm{cm}^{2} / \mathrm{V} \cdot \mathrm{s}\right): n_{e}=n j 10$ & $\mu\left(\mathrm{cm}^{2} / \mathrm{V} \cdot \mathrm{s}\right): n_{e}=n j 100$ \\
\hline 0 & $9.8 \times 10^{3}$ & & \\
\hline $1 \times 10^{15}$ & $7.6 \times 10^{3}$ & $7.6 \times 10^{3}$ & \\
\hline $1 \times 10^{16}$ & $7.2 \times 10^{3}$ & $7.2 \times 10^{3}$ & $6.0 \times 10^{3}$ \\
\hline $1 \times 10^{17}$ & $5.4 \times 10^{3}$ & $4.3 \times 10^{3}$ & $3.3 \times 10^{3}$ \\
\hline $1 \times 10^{18}$ & $3.0 \times 10^{3}$ & $1.7 \times 10^{3}$ & $1.1 \times 10^{3}$ \\
\hline
\end{tabular}

Table 3: Low field mobility in GaAs $(T=300 \mathrm{~K})$, as a function of ionized impurity density, $n_{i}$, and electron density, $n_{e}$ 


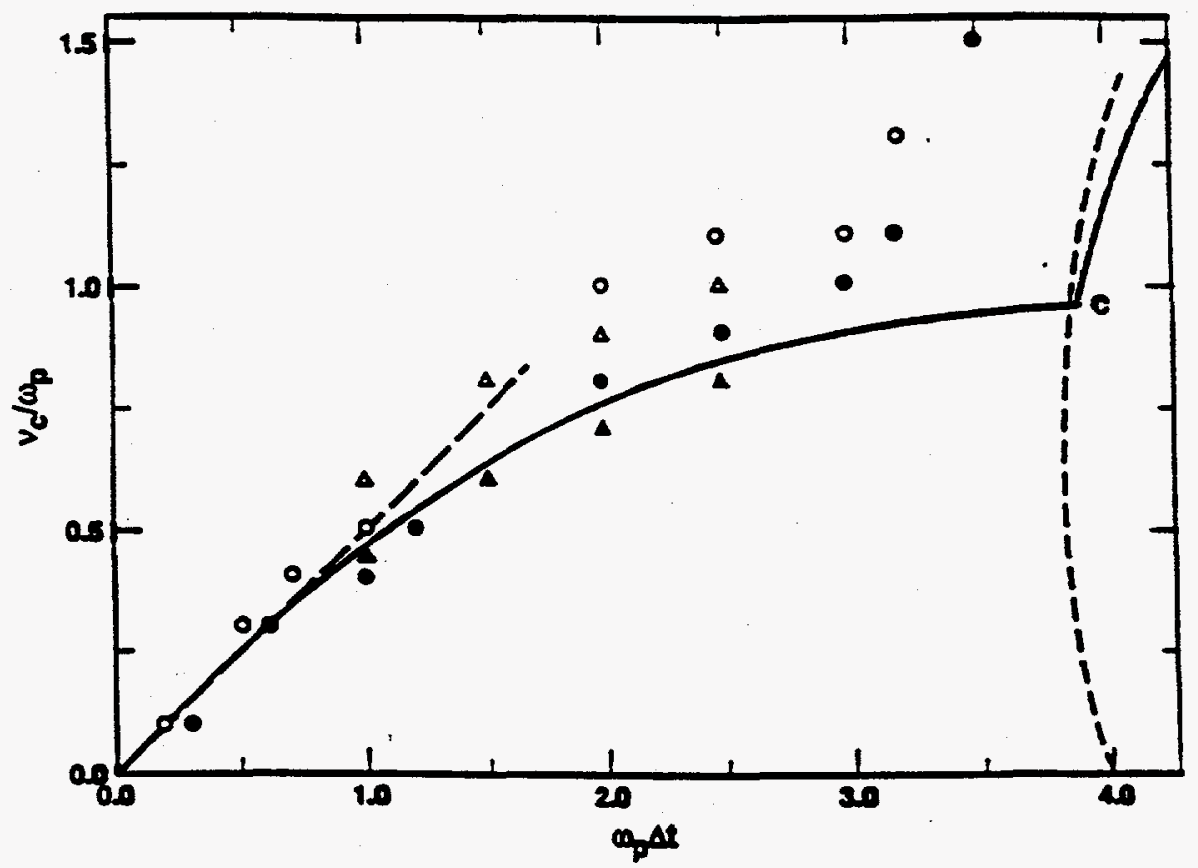

Fig. 1. Numerical stability as a function of collisionality, $v_{c} / \omega_{p}$, and time step between Poisson solves, $\omega_{p} \Delta t$. Markers represent results of simulations: open for stable, closed for unstable.

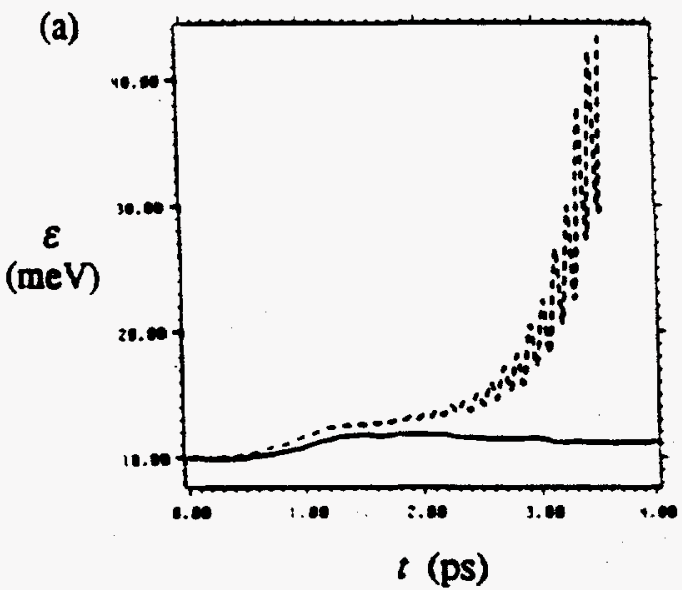

(b)

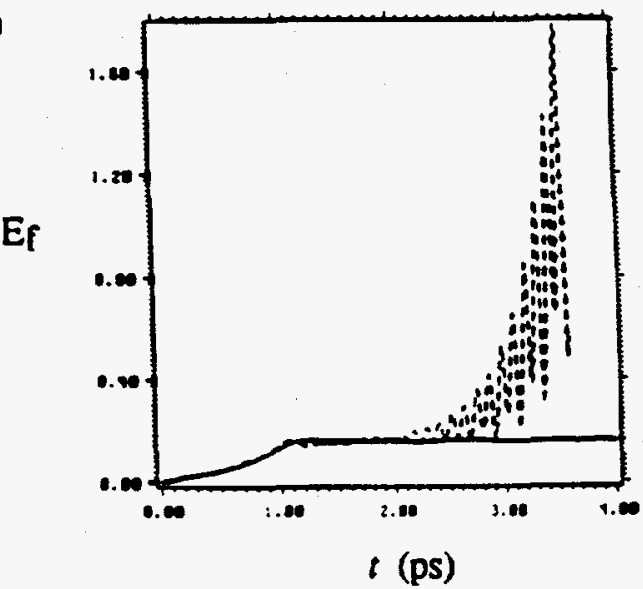

Fig. 2. Time history of (a) average particle energy and (b) electrostatic field energy (arbitrary units) from two diode calculations: explicit $\omega_{p} \Delta t=0.42$ (dashed line) and implicit $\omega_{p} \Delta t=2.24$ (solid line). 


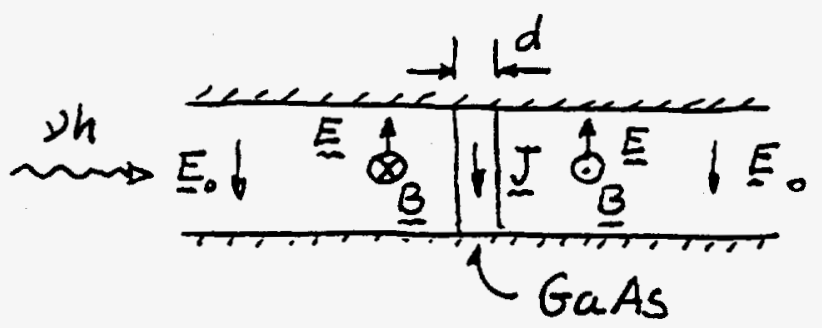

Fig. 3 Schematic of a photoswitch in a parallel-plate transmission line

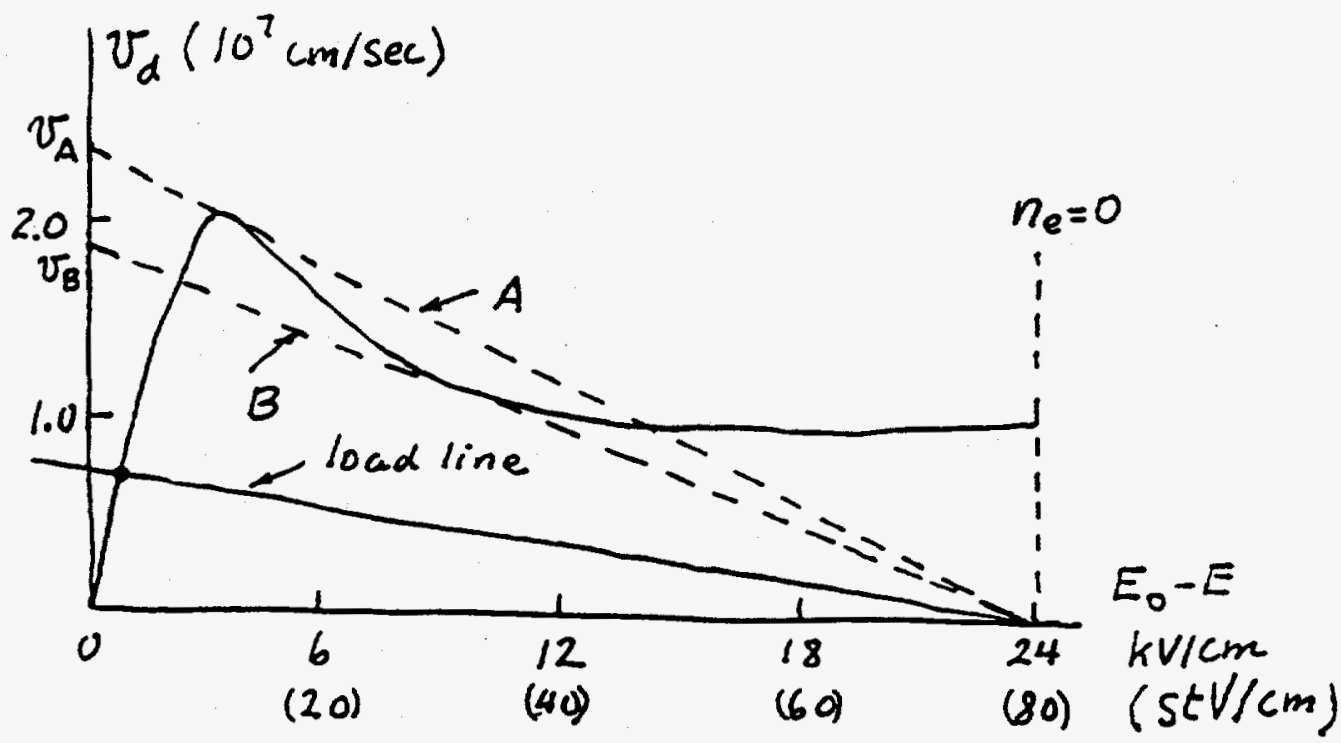

Fig. 4 Drift velocity vs. electric field for GaAs showing limiting load lines A and B defining saturated and unsaturated regimes. 


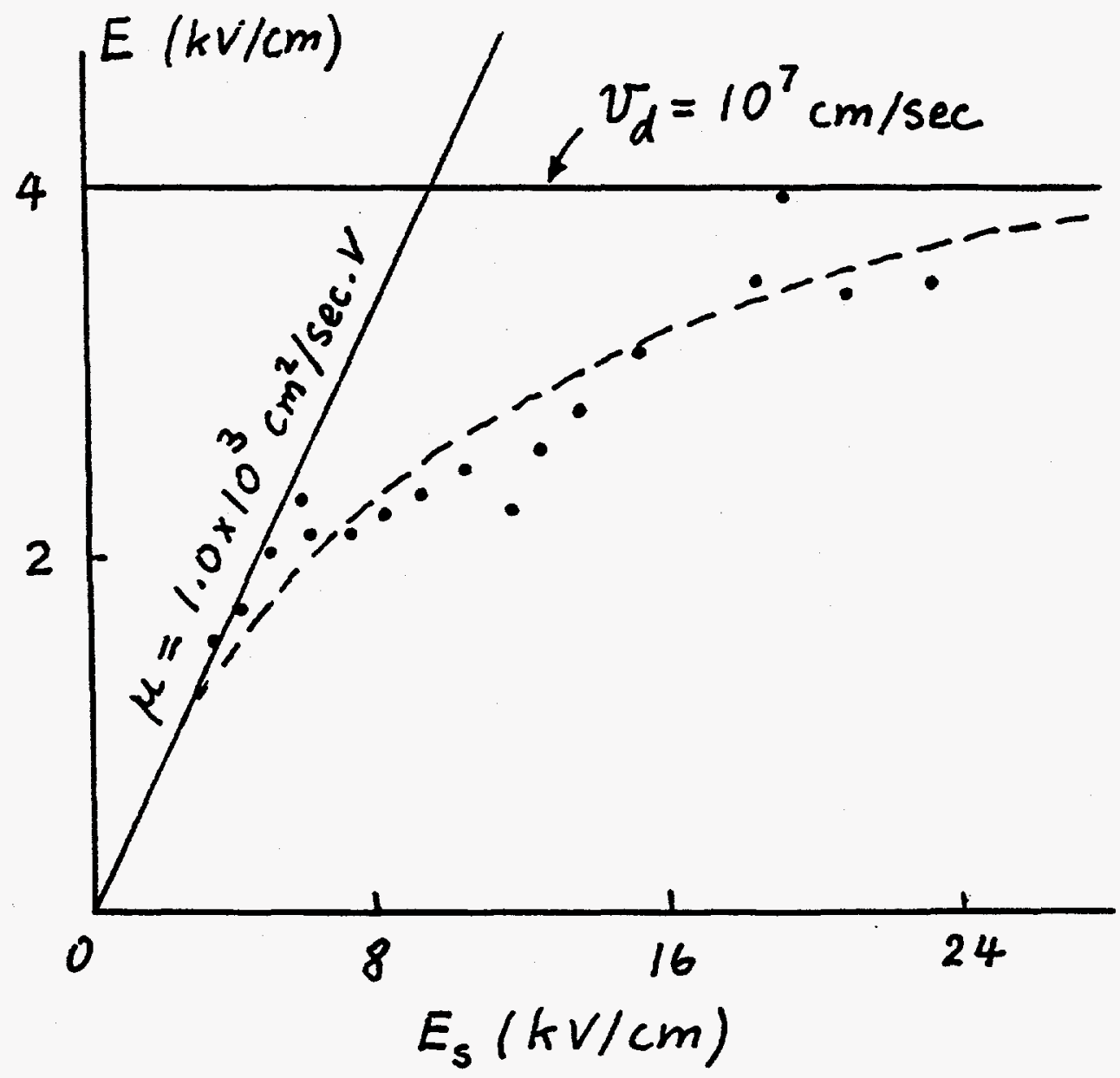

Fig. 5(a) Data from Fig. 9(b) of Ref. 6, replotted to show drift velocity characteristics.

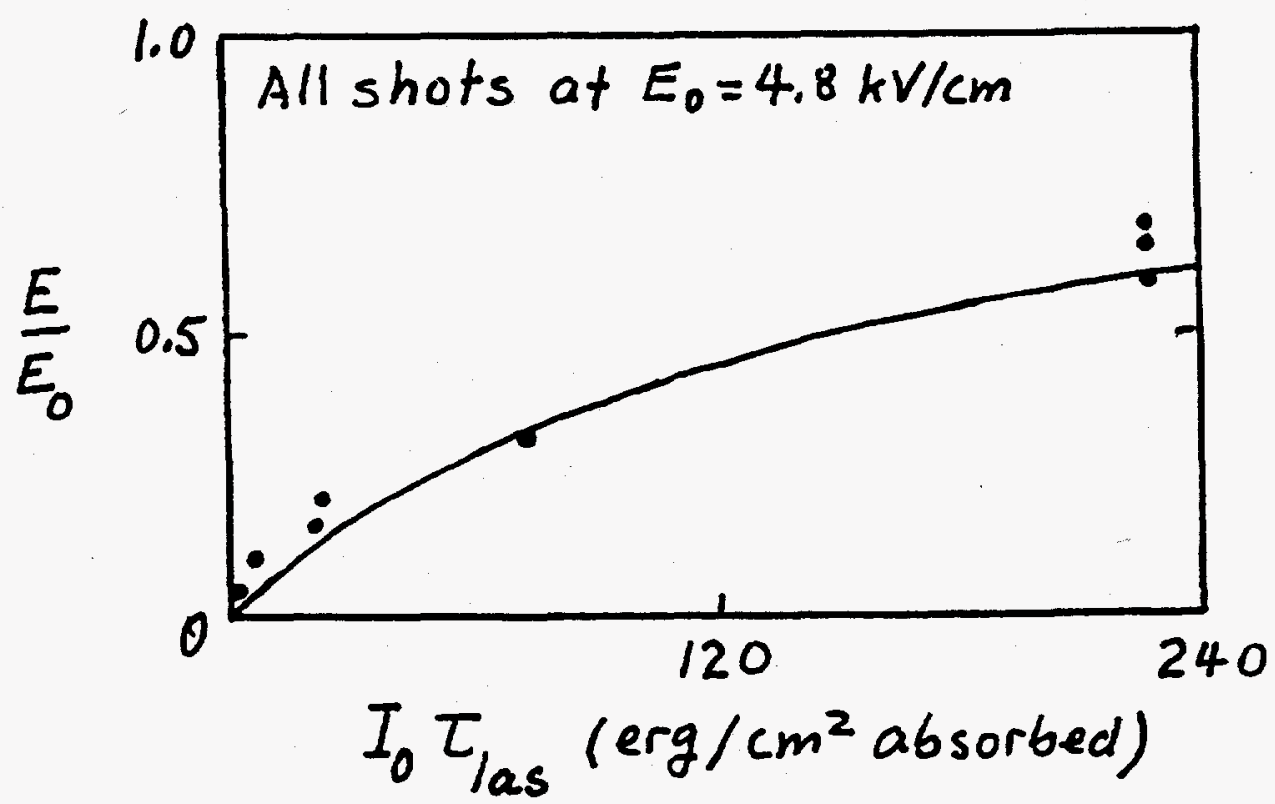

Fig. 5(b) Data from Fig. 9(a) of Ref. 6, plotted with Eq. (2.5) from Section 2; atext describes evaluation of necessary parameters. 


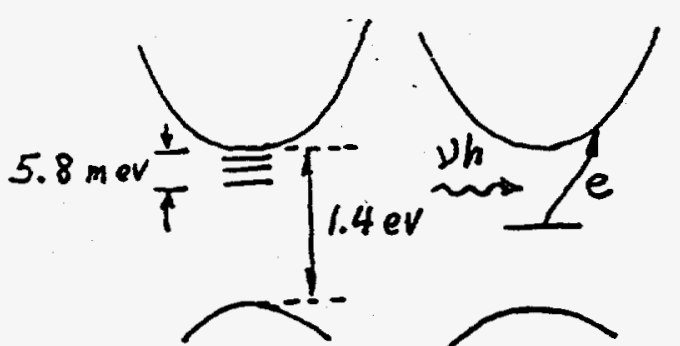

(a)

(b)

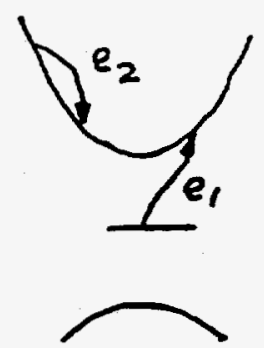

(c)

Fig. 6: Donor impurities; (a) Shallow impurity showing excited states; (b) Photoionization of a deep-level impurity; (c) Electron impact ionization.

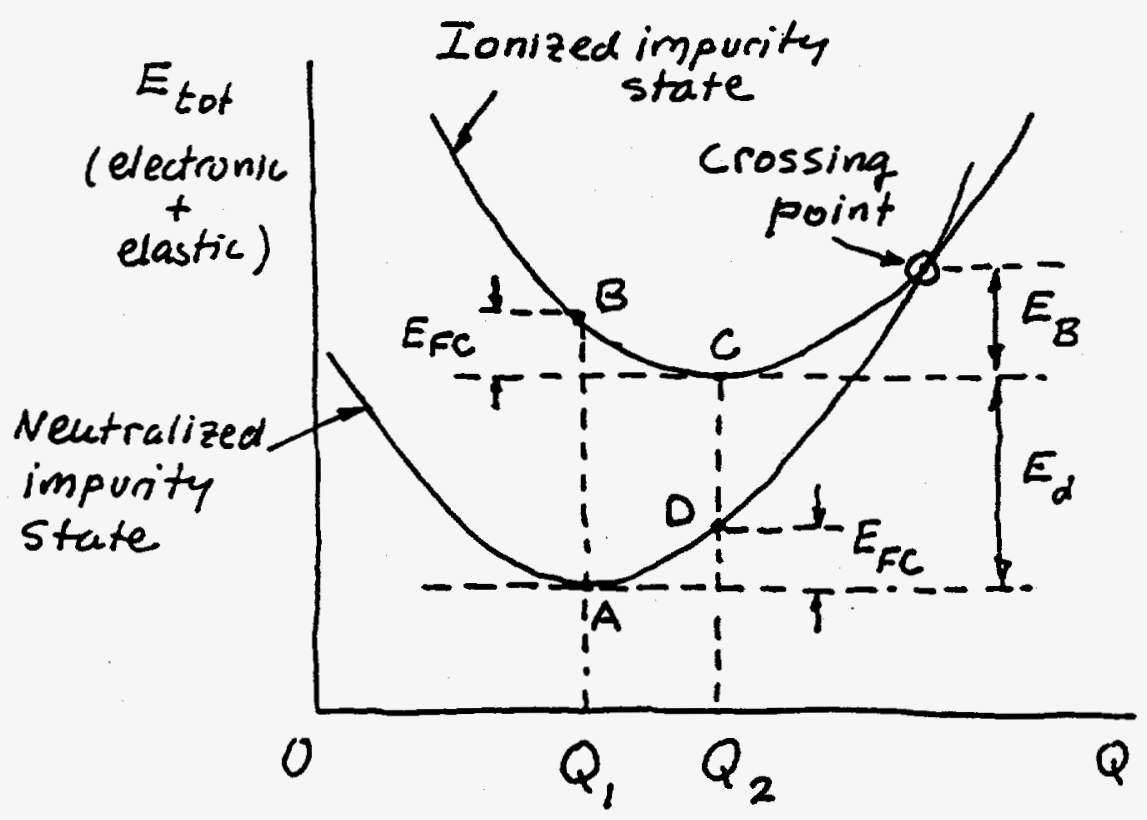

Fig. 7: Configuration coordinate diagram for multiphonon ionization and capture. 


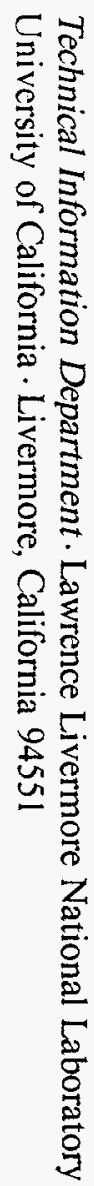

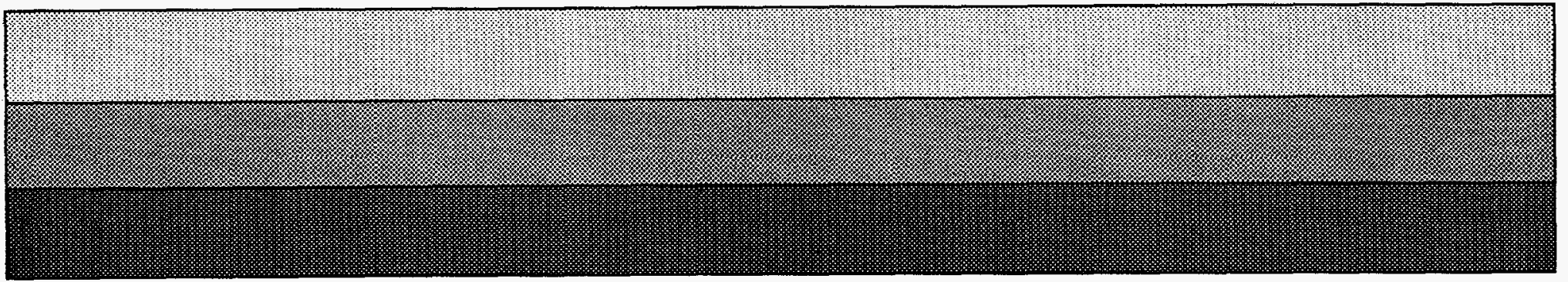

